\title{
Few-Photon Multiple Ionization of Ne and Ar by Strong Free-Electron-Laser Pulses
}

\author{
R. Moshammer, ${ }^{1}$ Y. H. Jiang, ${ }^{1}$ L. Foucar, ${ }^{2}$ A. Rudenko, ${ }^{1}$ Th. Ergler, ${ }^{1}$ C. D. Schröter, ${ }^{1}$ S. Lüdemann, ${ }^{1}$ K. Zrost, ${ }^{1}$ D. Fischer, ${ }^{3}$ \\ J. Titze, ${ }^{2}$ T. Jahnke, ${ }^{2}$ M. Schöffler, ${ }^{2}$ T. Weber, ${ }^{2,4}$ R. Dörner, ${ }^{2}$ T. J. M. Zouros,${ }^{5,6}$ A. Dorn, ${ }^{1}$ T. Ferger, ${ }^{1}$ K. U. Kühnel, ${ }^{1}$ \\ S. Düsterer ${ }^{4}$ R. Treusch, ${ }^{4}$ P. Radcliffe, ${ }^{4}$ E. Plönjes, ${ }^{4}$ and J. Ullrich ${ }^{1, *}$ \\ ${ }^{1}$ Max-Planck-Institut für Kernphysik, Saupfercheckweg 1, 69117 Heidelberg, Germany \\ ${ }^{2}$ Institut für Kernphysik, Universität Frankfurt, D 60486 Frankfurt, Germany \\ ${ }^{3}$ Atomic Physics, Stockholm University, Alba Nova University Centrum, 10691 Stockholm, Sweden \\ ${ }^{4}$ DESY, Notkestrasse 85, 22607 Hamburg, Germany \\ ${ }^{5}$ Department of Physics, University of Crete, P.O. Box 2208, 71003 Heraklion, Crete, Greece \\ ${ }^{6}$ Institute of Electronic Structure and Laser, P.O. Box 1527, 71110 Heraklion, Crete, Greece
}

(Received 5 February 2007; published 14 May 2007)

\begin{abstract}
Few-photon multiple ionization of $\mathrm{Ne}$ and Ar atoms by strong vacuum ultraviolet laser pulses from the free-electron laser at Hamburg was investigated differentially with the Heidelberg reaction microscope. The light-intensity dependence of $\mathrm{Ne}^{2+}$ production reveals the dominance of nonsequential two-photon double ionization at intensities of $I<6 \times 10^{12} \mathrm{~W} / \mathrm{cm}^{2}$ and significant contributions of three-photon ionization as $I$ increases. $\mathrm{Ne}^{2+}$ recoil-ion-momentum distributions suggest that two electrons absorbing "instantaneously" two photons are ejected most likely into opposite hemispheres with similar energies.
\end{abstract}

Single-photon-induced multiple ionization or excitation of atoms and molecules has been central to atomic and molecular physics research due to their prototype character to explore electron-electron correlation. Experimental milestones were, among others, kinematically complete measurements on double ionization of $\mathrm{He}$ and $\mathrm{H}_{2}$ (see, e.g., $[1,2])$, the fundamental two-electron systems. Here quantum mechanical $a b$ initio calculations have emerged since 1998, with predictions being in excellent agreement with all available data such that single-photon double ionization of $\mathrm{He}$ is considered to be understood [3] within the validity of the dipole approximation. Multiphotoninduced multiple ionization, at the other extreme, where a couple of ten photons are typically needed using lasers in the visible, has until the present day resisted any comprehensive theoretical description due to tremendous complications in solving the nonperturbative, few-particle quantum problem (see, e.g., [4]).

Few-photon multiple ionization, e.g., the interaction of two or three photons with two or more electrons, represents one of the most fundamental nonlinear processes, bridges the gap between the single- and multiphoton regimes, and, thus, is of decisive importance to advance nonlinear theories. Mainly due to its perturbative nature at shorter wavelengths along with the fact that only a few photons are involved, full quantum calculations are at the horizon [5].

The free-electron laser at Hamburg (FLASH) [6] delivering vacuum ultraviolet (VUV) photons at unprecedented intensities, in combination with the most advanced multiparticle detection systems, such as the Heidelberg reaction microscope [7], open the door for performing kinematically complete experiments in this new regime. At not too high intensities $\left(I \approx 10^{13} \mathrm{~W} / \mathrm{cm}^{2}\right)$, multiphoton ionization (MPI) can be expected to be the dominant process for many-electron removal. MPI contains two basic dynamical processes: an indirect two-step, "sequential ionization" (SI) mechanism and the direct one-step, "nonsequential ionization" (NSI, here we adopt the frequently used SI, NSI terminology). NSI requires that electrons are ionized by simultaneous absorption of photons (not necessarily coherent), whereas SI is a sequential absorption process within the same light pulse from FLASH. Since for SI the intermediate ionic state populated after the first, independent ionization step is usually assumed to be relaxed in its electronic ground state before further photons are absorbed, typically more photons are needed than for NSI to reach the same final charge state. NSI, on the other hand, due to its simultaneous rather than "step-by-step" character, provides a much richer setting for photon-electron interaction as well as electron-electron correlation mechanisms, making it the more exciting process.

Differential experimental data for MPI represent the most critical testing ground for theories. Besides the basic character of such experiments, there is tremendous practical interest since these nonlinear processes govern the interaction of the VUV radiation with matter in general and have to be understood if more complicated reactions with surfaces, bulk matter, or biological samples shall be investigated.

Few experiments on total cross sections have been reported so far. Recently, two-photon single ionization of $\mathrm{He}$ was investigated by Laarmann et al. [8] with FLASH radiation at the $98 \mathrm{~nm}$ wavelength. Nabekawa et al. [9] observed the production of doubly charged He ions by twophoton absorption using $42 \mathrm{eV}$ high-harmonic radiation. Again at $98 \mathrm{~nm}$ with FLASH, Wabnitz et al. [10] studied multiple ionization of $\mathrm{Ar}$ and $\mathrm{Xe}$ atoms, and experimentally determined multiply charged $\mathrm{Xe}^{q+}$ yields were inter- 
preted in terms of the absorption of several VUV photons with the help of an independent-particle model [11]. Here $q$ denotes the charge state of the ion. Very recently, Benis et al. [12] observed two-photon double ionization of $\mathrm{Ar}$ and $\mathrm{Kr}$ atoms by a superposition of harmonics.

In this Letter, we present momentum-resolved experimental spectra for two- and three-photon double ionization of Ne obtained with a reaction microscope at FLASH. The data provide first information about the sum momentum of two emitted electrons supporting dominant NSI two-photon contributions at light intensities of a few $10^{12} \mathrm{~W} / \mathrm{cm}^{2}$ in accordance with the recorded intensity dependence of $\mathrm{Ne}^{2+}$ ion yields. With increasing intensity, three-photon processes are found to become more and more important.

Our reaction microscope was installed at the focus of beam line BL 2 at FLASH. The light at $32 \mathrm{~nm}(\approx 38.8 \mathrm{eV})$, with a $5 \mathrm{~Hz}$ repetition rate at pulse energies $\approx 1-10 \mu \mathrm{J}$, was focused into the interaction chamber reaching peak intensities of $I \approx 10^{12}-10^{13} \mathrm{~W} / \mathrm{cm}^{2}$. The free-electronlaser (FEL) beam was crossed with a well-collimated ( $2 \mathrm{~mm}$ diameter), dilute $\left(\approx 10^{9}\right.$ particles $\left./ \mathrm{cm}^{3}\right)$, and intrinsically cold $\left(T_{\text {jet }} \approx 2 \mathrm{~K}\right)$ supersonic atomic gas jet. Ions produced by the interaction with the FEL pulse were projected by means of a weak electric field $(0.24 \mathrm{~V} / \mathrm{cm})$ onto time- and position-sensitive microchannel plate detectors (diameter $120 \mathrm{~mm}$, position resolution $0.1 \mathrm{~mm}$ ). From the measured time of flight and position of each individual particle, the initial 3-dimensional momentum vectors were reconstructed, and an ion-momentum resolution of $\approx 0.8$ a.u. for $\mathrm{Ne}^{+}$was achieved (for details, see [7]).

The current induced on a metal plate by each individual FLASH pulse was recorded in order to obtain pulse-topulse information of the respective pulse energy. By comparing the average induced current over several thousand pulses with the mean pulse energy given from the machine, the absolute energy of each pulse could be deduced. On the basis of this number, the approximately known focal spot size (30 $\mu \mathrm{m}$ diameter), and the pulse duration (30 fs), $I$ was calculated with an estimated uncertainty of a factor of 5. The linearity of our intensity scale was assured to be correct within $10 \%$ with a relative calibration between runs of better than $50 \%$ by comparing different yields for single ionization $\left({ }^{20} \mathrm{Ne}^{+},{ }^{22} \mathrm{Ne}^{+}, \mathrm{H}_{2} \mathrm{O}^{+}\right.$, etc. $)$as a function of $I$.

The time-of-flight spectra of $\mathrm{Ne}^{q+}$ for $q=1$ and 2, and $\operatorname{Ar}^{q+}$ for $q=1-3$, are shown in Fig. 1. The spectrum is dominated by peaks of singly charged ${ }^{20} \mathrm{Ne}^{+}$and ${ }^{22} \mathrm{Ne}^{+}$ ions with small $(\sim 2 \%)$ admixtures from the residual ion $\mathrm{H}_{2} \mathrm{O}_{+}$(broad hump). In addition, small but significant yields of ${ }^{20} \mathrm{Ne}^{2+}$ ions were observed. Similarly to $\mathrm{Ne}$, MPI of Ar producing up to $\mathrm{Ar}^{3+}$ was found (Fig. 1 inset).

In analyzing the data, one has to first clarify whether multiply charged $\mathrm{Ne}^{q+}$ and $\mathrm{Ar}^{q+}$ ions result from MPI or single-photon absorption of high-harmonic radiation, which was not actively suppressed in the present experiment. On the basis of the well-known cross sections for

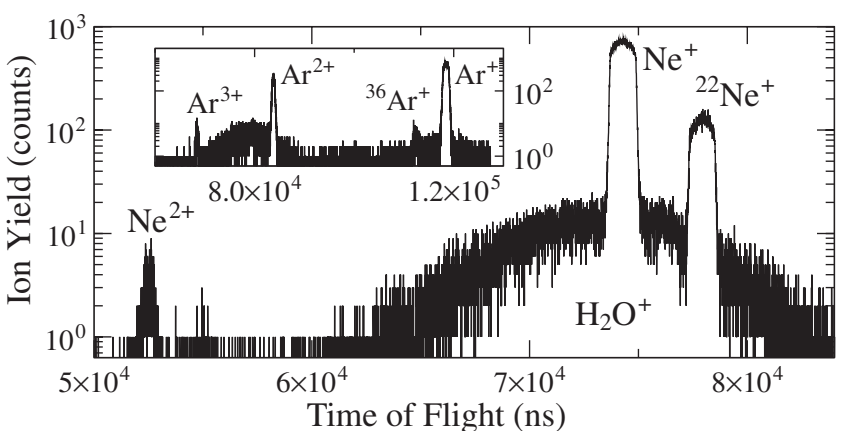

FIG. 1. Time-of-flight mass spectra of $\mathrm{Ne}^{(1-2)+}$ and $\mathrm{Ar}^{(1-3)+}$ ions (in the inset) at $I \approx 10^{12}-10^{13} \mathrm{~W} / \mathrm{cm}^{2}$.

single-photon single and double ionization of $\mathrm{Ne}$ by synchrotron radiation $[13,14]$, one can estimate the ionizationrate ratio $P_{\mathrm{Ne}^{2+}} / P_{\mathrm{Ne}^{+}}$to be $\approx 7.5 \times 10^{-5}$ for single-photon absorption from the second and third harmonics. For this estimate, the relative intensity ratios of the second and third harmonics to the fundamental were taken from Ref. [15] in the $10-40 \mathrm{~nm}$ range to be $2 \mathrm{nd} / 1 \mathrm{st}=0.35 \%$ and $3 \mathrm{rd} / 1 \mathrm{st}=0.4 \%$, respectively. This result is in contrast to the measured ratios of $2.6 \times 10^{-4}$ to about $2 \times 10^{-3}$, providing evidence that the observed multicharged ions result mainly from few-photon ionization.

The first and second ionization potentials for $\mathrm{Ne}$ are 21.6 and $40.9 \mathrm{eV}$, respectively, and the first four ionization potentials of Ar are 15.8, 27.7, 40.7, and 59.8 eV. Therefore, a minimum energy of $62.5 \mathrm{eV}$ is needed to doubly ionize $\mathrm{Ne}$ atoms, which means that at least 2 photons have to be absorbed at the present photon energy of $38.8 \mathrm{eV}$. Certainly, with much lower probability though, more than two photons can be absorbed. This would, according to perturbation theory, most likely be SI. Here, within one laser pulse, the $\mathrm{Ne}$ atom gets first singly ionized by absorption of one photon, and then, in a further step, a second electron is removed from the relaxed $\mathrm{Ne}^{+}$ground state, requiring additional two photons. Note that whereas the lowest $\mathrm{Ne}^{+}$excited state cannot be reached with one photon from the neutral Ne ground state, the two-photon ionization of $\mathrm{Ne}^{+}$can be enhanced via an intermediate resonance. If in total three photons are involved, we cannot distinguish between SI and NSI (this would require one to measure electron energy spectra in addition), and, thus, we just use the terminology three-photon double ionization (3PDI). In any case, according to lowest-order perturbation theory, expected to be valid at $I<10^{15} \mathrm{~W} / \mathrm{cm}^{2}$ [16], the ionization yield should increase with $I$ as $Y=\sigma_{n} \cdot I^{n}$, where $\sigma_{n}$ is the generalized $n$-photon cross section and $n$ is the number of photons needed for ionization.

The $\mathrm{Ne}^{2+}$ yield is analyzed as a function of $I$ in Fig. 2 . The blue and red curves, fitted to two sets of data, nicely describe the experimental intensity dependences of $\mathrm{Ne}^{2+}$ ions with the slopes $n=2.2 \pm 0.2$ and $n=2.6 \pm$ 0.2 , respectively. This indicates that double ionization of $\mathrm{Ne}$ is induced mainly via a two-photon absorption at 


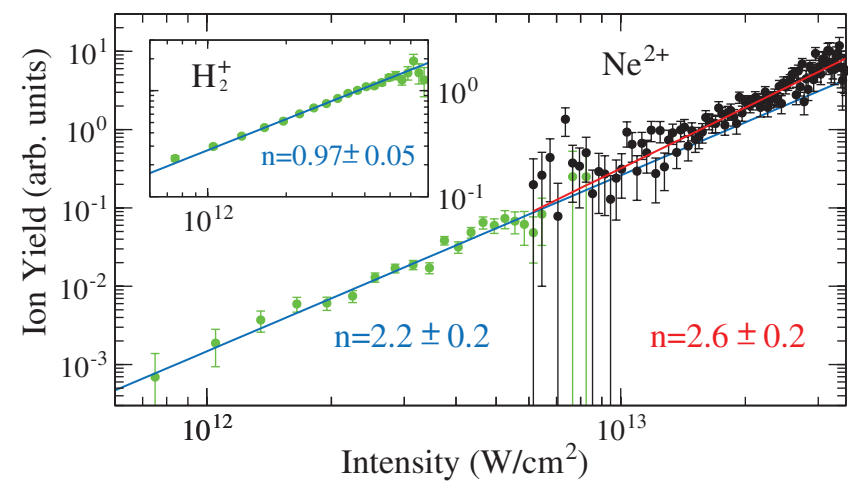

FIG. 2 (color). Ion yield of $\mathrm{Ne}^{2+}$ and $\mathrm{H}_{2}^{+}$(inset) as a function of $I$. Solid black and green points are the present data for two different sizes of the light collimation exit slit. Solid blue and red lines are fits by $\log Y=n \log I+\log \sigma_{n}$ to two sets of data yielding $n=2.2 \pm 0.2$ and $n=2.6 \pm 0.2$. Single ionization of $\mathrm{H}_{2}$ residual gas leading to the slope $n=0.97 \pm 0.05$ provides further evidence for the linearity of our intensity scale.

$I<6 \times 10^{12} \mathrm{~W} / \mathrm{cm}^{2}$, clearly favoring the NSI mechanism. The slope $n=2.6$ found at higher intensities points to mixed NSI and 3PDI transition contributions. As I increases, the contribution of three-photon absorption is expected to dominate [17]. The ionization-rate ratio $P_{\mathrm{Ne}^{2+}} / P_{\mathrm{Ne}^{+}}$agrees well with the measurements taken at the same photon energy by Sorokin et al. [18].

Similar conclusions can be drawn from the $\mathrm{Ar}^{3+}$ intensity dependence shown in Fig. 3(b). The slopes deliver the values $n=2.0 \pm 0.1$ and $n=3.5 \pm 0.2$ for $\mathrm{Ar}^{2+}$ and $\mathrm{Ar}^{3+}$, respectively. According to the total ionization potentials for double and triple ionization, $\mathrm{Ar}^{3+}$ can be created by three (NSI) or four (SI) photons, and two photons are needed for $\mathrm{Ar}^{2+}$ production. Analogous to the Ne data at high intensities, $n=3.5$ indicates that three- and fourphoton absorptions comparably contribute to the creation of $\mathrm{Ar}^{3+}$. Since the same number of photons is needed to ionize $\mathrm{Ar}^{2+}$ for NSI as well as SI, the value $n=2.0 \pm 0.1$ further confirms the reliability of the measured intensities and ionization rates. Our finding may not completely agree with recent results [10], where sequential ionization was observed to be the dominant mechanism for MPI of Ar and

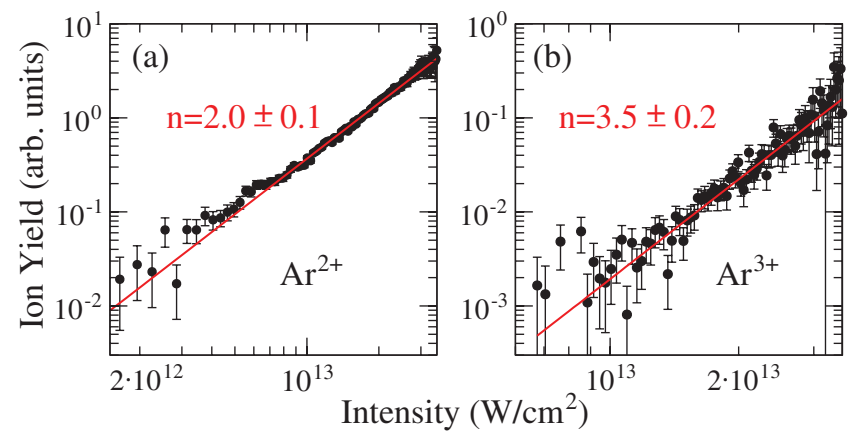

FIG. 3 (color). Yield of $\mathrm{Ar}^{q+}$ ions as a function of $I$, (a) $q=2$ and (b) $q=3$; for details, see the caption of Fig. 2.
$\mathrm{Xe}$ at comparable intensities but lower photon energies $(12.7 \mathrm{eV})$.

In addition to the total ionization yield, we were able to record the first recoil-ion-momentum distribution for twophoton double ionization of Ne in Fig. 4. Shown are the ion-momentum components in the $x-y$ plane for various integration intervals along $z$ as stated in the figure caption. The light-propagation direction and its polarization are along $z$ and $y$, respectively. In Fig. 4(a), the recoil-ionmomentum distribution of $\mathrm{Ne}^{+}$is observed to cluster on a circle with the radius $P_{r}=\left(P_{x}^{2}+P_{y}^{2}\right)^{1 / 2}=1.05$ a.u. as expected for absorption of one single photon. Ions produced by two-photon or second-harmonic photon absorption are clearly absent, expected to occur at the large circle at $P_{r}=2.0$ a.u. in Fig. 4(a). This result, obtained with a statistical significance of about $10^{-2}$ relative to one-photon single ionization, agrees with the measured ion-yield dependence in Fig. 2.

The $\mathrm{Ne}^{2+}$ pattern, representing the vector summomentum distribution of the two electrons, is shown in Figs. 4(b) and 4(c) for $I<3 \times 10^{12} \mathrm{~W} / \mathrm{cm}^{2}$ and $I>2 \times$ $10^{13} \mathrm{~W} / \mathrm{cm}^{2}$, respectively. For NSI, the electron excess energy is $\cong 15 \mathrm{eV}$, resulting in $P_{r} \leq 1.5$ a.u. indicated as circle 2 in Figs. 4(b) and 4(c). For absorption of three photons, the maximum two-electron sum momentum (emission into the same direction) can be 2.76 a.u., plotted as circle 3. Denoting $C_{i}$ to represent the numbers of events inside the circle $i(i=1,2,3)$, we might draw conclusions about the contribution of various processes. Thus, the rate $\left(C_{3}-C_{2}\right) / C_{3}$ of events in Fig. 4(b) point to a $29 \%$ contribution of three-photon absorption and a $C_{2} / C_{3}=71 \%$ contribution of NSI inside circle 2 . The observation of both two- and three-photon absorption in the ion-momentum spectra at $I<3 \times 10^{12} \mathrm{~W} / \mathrm{cm}^{2}$ is in qualitative agreement with the intensity dependence presented in Fig. 2. The two-photon NSI is certainly the dominant transition process while three-photon DI, most likely SI, is active simultaneously.

For higher light intensities, i.e., at $I>2 \times 10^{13} \mathrm{~W} / \mathrm{cm}^{2}$, $\left(C_{3}-C_{2}\right) / C_{3}$ increases to a $35 \%$ contribution, whereas $C_{2} / C_{3}$ slightly decreases to $65 \%$, a clear indication that the SI contribution increases with $I$, which supports the findings in the intensity dependence above. Note that, due to present momentum resolution $(\approx 0.8$ a.u. $)$ and overlapping emission patterns for two- and three-photon processes, it is impossible to absolutely separate these two transition mechanisms by measuring recoil-ion momenta alone. Thus, $C_{3}-C_{2}$ could also include some events of NSI and vice versa.

Circle 1 in Figs. 4(b) and 4(c) with a radius of 1.05 a.u. contains the events for two electrons ejected most likely into opposite hemispheres assuming similar energies and amounts to $C_{1} / C_{2}=66 \%$ of the NSI contribution in Fig. 4(b). For two-photon double ionization, the angular momentum constraints for the relative emission of the two electrons are much more relaxed as compared to single- 


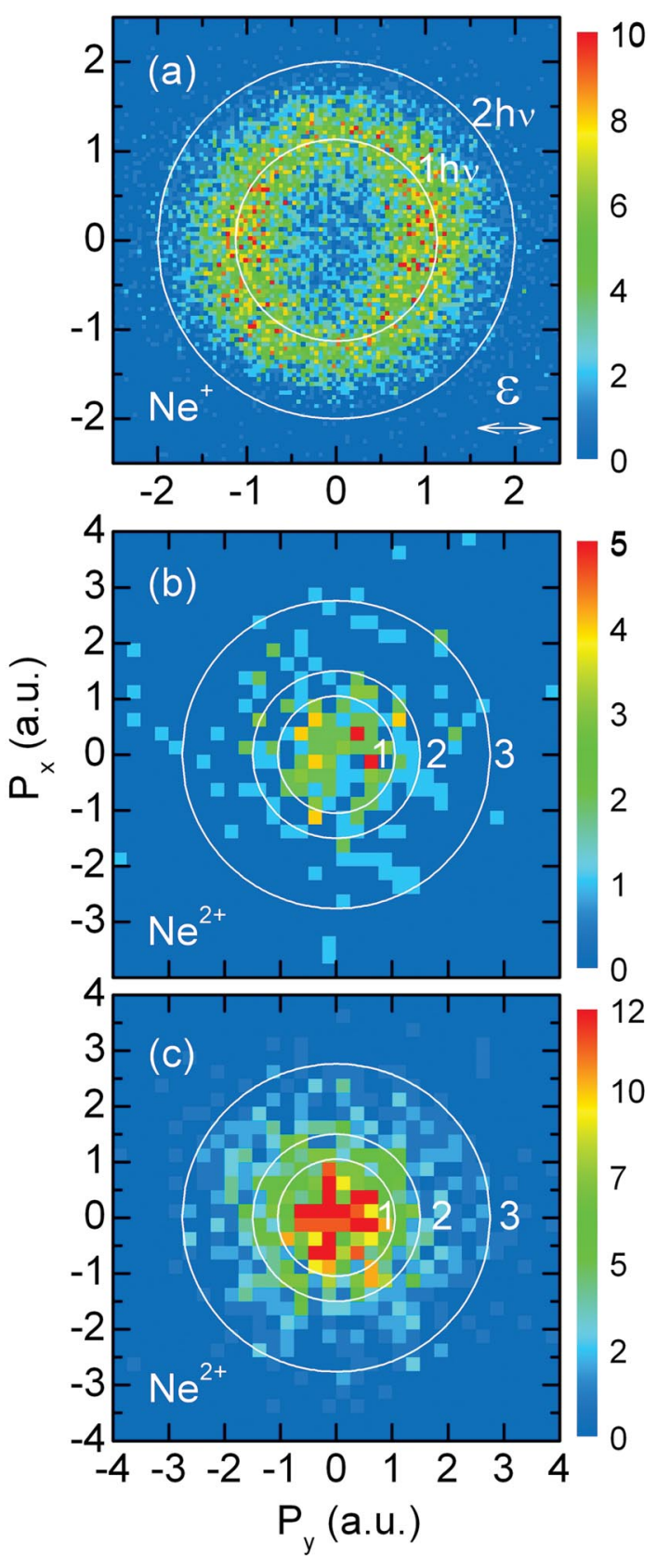

FIG. 4 (color). Density plot of recoil-ion-momentum distributions for (a) $\mathrm{Ne}^{+}$and (b), (c) $\mathrm{Ne}^{2+}$. The solid curves indicate the momentum positions for one- and two-photon absorption in (a) as well as for NSI and three-photon processes in (b) and (c) for various emission mechanisms; see text. $\mathrm{Ne}^{+}$momenta are integrated over events with $\left|P_{z}\right| \leq 0.1$ a.u. at low intensities corresponding to the green points in Fig. 2. $\mathrm{Ne}^{2+}$ distributions integrate all events along the $z$ direction at (b) $I<$ $3 \times 10^{12} \mathrm{~W} / \mathrm{cm}^{2}$ and (c) $I>2 \times 10^{13} \mathrm{~W} / \mathrm{cm}^{2}$. The polarization of the light is along the $y$ axis.

photon double ionization. The observed pattern shows a clear maximum at zero momentum corresponding to a kinematics where both electrons are emitted with similar energies into roughly opposite directions. For singlephoton double ionization, this configuration is suppressed and even forbidden for equal energy sharing if the $\mathrm{Ne}^{2+}$ is in the electronic ground state (even symmetry). Singlephoton double ionization of $\mathrm{He}$, depicted in Fig. 1 of Ref. [19], shows a pronounced double-hump structure in the recoil-ion-momentum distribution, reflecting the dipole emission characteristics as a result of the angular momentum selection rules, in clear disagreement with the present findings. Back-to-back emission for NSI supports theoretical predictions for He [20], where this geometry was found to represent the main decay channel.

In summary, we studied few-photon multiple ionization of $\mathrm{Ne}$ and Ar using FLASH photons in the VUV regime. The light-intensity dependence for ion yields confirmed double ionization of $\mathrm{Ne}$ to be mainly due to two-photon nonsequential ionization at $I<6 \times 10^{12} \mathrm{~W} / \mathrm{cm}^{2}$. As $I$ increases, three- or more-photon processes, most likely SI transitions for $\mathrm{Ne}^{2+}$ as well as $\mathrm{Ar}^{3+}$ production, become more pronounced. Recoil-ion-momentum distributions of $\mathrm{Ne}^{2+}$ confirm these observations and, for the first time, provide evidence for a sizable contribution of events, where two ejected electrons widely share the excess energy available and, thus, are emitted with similar energies, into back-to-back directions. With the increase of the FLASH pulse repetition rate achieved recently, fully differential measurements are deeming at the horizon.

Support from the Max-Planck-initiative for FEL studies and from HGF "Virtual Institut Atomic and Cluster Physics at FEL" is gratefully acknowledged. We are greatly indebted to the scientific [6] and technical team at FLASH, in particular, the machine operators and run coordinators, all together being the foundation of its outstanding performance.

*Electronic address: Joachim.Ullrich@mpi-hd.mpg.de

[1] O. Schwarzkopf et al., Phys. Rev. Lett. 70, 3008 (1993).

[2] Th. Weber et al., Nature (London) 431, 437 (2004).

[3] J. Briggs and V. Schmidt, J. Phys. B 33, R1 (2000).

[4] A. Becker and F. H. M. Faisal, J. Phys. B 38, R1 (2005).

[5] J. S. Parker et al., Phys. Rev. Lett. 96, 133001 (2006).

[6] V. Ayvazyan et al., Eur. Phys. J. D 37, 297 (2006).

[7] J. Ullrich et al., Rep. Prog. Phys. 66, 1463 (2003).

[8] T. Laarmann et al., Phys. Rev. A 72, 023409 (2005).

[9] Y. Nabekawa et al., Phys. Rev. Lett. 94, 043001 (2005).

[10] H. Wabnitz et al., Phys. Rev. Lett. 94, 023001 (2005).

[11] R. Santra and C. Creene, Phys. Rev. A 70, 053401 (2004).

[12] E. P. Benis et al., Phys. Rev. A 74, 051402(R) (2006).

[13] D. M. P. Holland et al., J. Phys. B 12, 2465 (1979).

[14] G. V. Marr and J. B. West, At. Data Nucl. Data Tables 18, 497 (1976).

[15] S. Düsterer et al., Opt. Lett. 31, 1750 (2006).

[16] M. Protopapas et al., Rep. Prog. Phys. 60, 389 (1997).

[17] P. Lambropoulos et al., Phys. Rev. A 72, 013410 (2005).

[18] A. Sorokin et al., Phys. Rev. A 75, 051402(R) (2007).

[19] A. Knapp et al., J. Phys. B 35, L521 (2002).

[20] S. X. Hu et al., J. Phys. B 38, L35 (2005). 